\title{
Lightwave control of the valley pseudospin in a monolayer of tungsten diselenide
}

\author{
Christoph P. Schmid ${ }^{1, *}$, Fabian Langer ${ }^{1}$, Stefan Schlauderer ${ }^{1}$, Martin Gmitra ${ }^{1}$, Jaroslav \\ Fabian $^{1}$, Philipp Nagler ${ }^{1}$, Christian Schüller ${ }^{1}$, Tobias Korn ${ }^{1}$, Peter G. Hawkins ${ }^{2}$, Johannes \\ T. Steiner ${ }^{2}$, Ulrich Huttner ${ }^{2}$, Stephan W. Koch ${ }^{2}$, Mackillo $\mathrm{Kira}^{3}$, and Rupert Huber ${ }^{1}$
}

\author{
${ }^{1}$ University of Regensburg, 93040 Regensburg, Germany \\ ${ }^{2}$ University of Marburg, 35032 Marburg, Germany \\ ${ }^{3}$ University of Michigan, Ann Arbor, Michigan 48109, USA
}

\begin{abstract}
As conventional electronic is approaching its ultimate limits, tremendous efforts have been taken to explore novel concepts of ultrafast quantum control. Lightwave electronics - the foundation of attosecond science - has opened a spectacular perspective by utilizing the oscillating carrier wave of an intense light pulse to control the translational motion of the electron's charge faster than a single cycle of light [1-7]. Despite their promising potential as future information carriers $[8,10]$, the internal quantum attributes such as spins and valley pseudospins have not been switchable at optical clock rates. Here we demonstrate a novel subcycle control scheme of the electron's pseudospin in a monolayer of tungsten diselenide using strong mid-infrared lightwaves [9]. Our work opens the door towards systematic valleytronic protocols at optical clock rates.
\end{abstract}

Steering the quantum motion of electrons in atoms and molecules by the carrier wave of an intense lightwave lies at the heart of high-harmonic generation and attosecond science [1]. Only recently this seminal concept of subcycle control of the electronic dynamics has been extended to solid-state system leading to the observation of high-harmonic generation [2-4], dynamical Bloch oscillations [3], quantum interference [7], and electron-hole collisions [67,9]. So far, however, solid-state lightwave electronics has mainly focused on controlling the translational motion of the electron's charge faster than a single cycle of light.

Despite their promising potential as information carriers, the internal degrees of freedom of solids such as spin or valley pseudospin have not been switched on a subcycle scale up to now. In particular, monolayers of transition metal dichalcogenides are an ideal material platform for such concepts $[4,8,10]$. These ultimately thin semiconductors feature two inequivalent band minima - called valleys - which can be selectively excited by circularly polarized light (Fig. 1b). It has already been shown that the coherence of the valley pseudospin is conserved in steady-state photoluminescence $[8,10]$ experiments rendering this spin-like quantity a promising candidate for an information bit. The subcycle manipulation of this degree of freedom, however, remained an open challenge.

\footnotetext{
*Corresponding author: christoph3.schmid@ur.de
} 

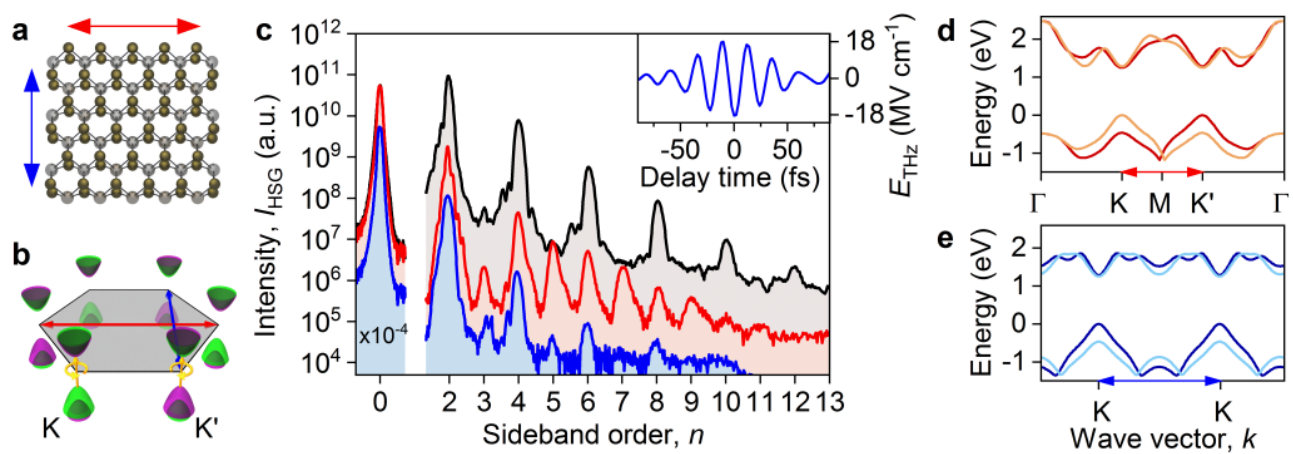

Fig. 1. a Crystal structure of a WSe 2 monolayer (tungsten atoms: grey spheres; selenium atoms: gold spheres). b Hexagonal Brillouin zone showing the spin-split valence and conduction bands at the high-symmetry K and $\mathrm{K}^{\prime}$ points. c High-order sideband spectra from bulk (black) and monolayer $\mathrm{WSe}_{2}$ in zigzag (red) and armchair (blue, shifted down by one order of magnitude) direction. Comparable intensities for even and odd orders are observed for the zigzag direction while the armchair direction shows no odd-orders. Inset: Electro-optically detected multi- $\mathrm{THz}$ transient. d, e, Band structure of monolayer $\mathrm{WSe}_{2}$ in the zigzag (red) and armchair (blue) direction as calculated by DFT (dark colors: spin-up; light colors: spin-down).

Here, we demonstrate a novel control scheme for the valley pseudospin by lightwavedriven intraband transport [9], which enables the implementation of valleytronic concepts at optical clock rates. To this end, we resonantly prepare coherent electron-hole pairs with a linearly polarized 100 -fs near-infrared pulse in bulk and monolayer $\mathrm{WSe}_{2}$. Simultaneously, an intense multi-THz waveform (inset Fig. 1c) ionizes the electron-hole pairs, accelerates the constituent electron and hole, and finally recollides them, analogously to the wellknown three-step model underlying attosecond pulse generation in atomic gases [1]. Upon recollision, electron and hole can recombine while emitting the kinetic energy imparted by the acceleration plus the bandgap energy via a photon.

In bulk $\mathrm{WSe}_{2}$, these dynamics lead to the emission of a sequence of high-order sidebands accompanying the excitation spectrum. As bulk $\mathrm{WSe}_{2}$ is inversion-symmetric, highorder sideband generation (HSG) is independent of the polarity of the driving field and collisions between electrons and holes occur for every half-cycle, which corresponds to a spectral modulation of the emitted sidebands with $2 v_{\mathrm{THz}}$ (Fig. 1c, black curve).

The first high-order sideband spectrum from a monolayer of $\mathrm{WSe}_{2}$ (Fig. 1c, red curve) differs qualitatively from the bulk case showing even and odd order sidebands of comparable strength. Despite the ultimately low thickness of the monolayer, sidebands up to the order of $n=11$ are observed. The strongest odd-order sideband intensity occurs when electron-hole pairs are accelerated along the zigzag crystal direction (Fig. 1c, red curve) whereas a THz field polarized along the armchair direction leads to a suppressed HSG (Fig. $1 \mathrm{c}$, blue curve). Since inversion symmetry along the zigzag direction is only broken in momentum space (Fig. 1d, red arrow), not in real space (Fig. 1a, red arrow), the emergence of odd-order sidebands must be related with intraband transport. In the zigzag direction the electron-hole pairs experience strong asymmetries of the band structure for large excursions from the K or K' point, supporting odd-order HSG (see Fig. 1d). In contrast, the band structure in the armchair direction is symmetric about the $\mathrm{K}$ and the $\mathrm{K}$ ' points (see Fig. 1e) leading to a suppressed emission of odd order sidebands.

This explanation, however, is incomplete as the linearly-polarized excitation pulse populates both valleys equally. Due to the mirror symmetry of the bands with respect to the $\mathrm{K}$ and $\mathrm{K}$ ' points, the electron-hole dynamics for positive and negative $\mathrm{THz}$ fields are symmetric, which forbids odd-order HSG. This contradiction can be resolved if the valley pseudospin is taken into account. This spin-like quantity connects emission from different valleys with circularly polarized light of opposite helicity. This effectively breaks the 


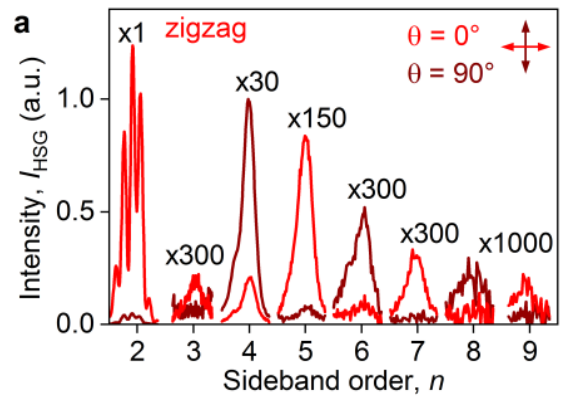

b Polarization angle, $\theta$

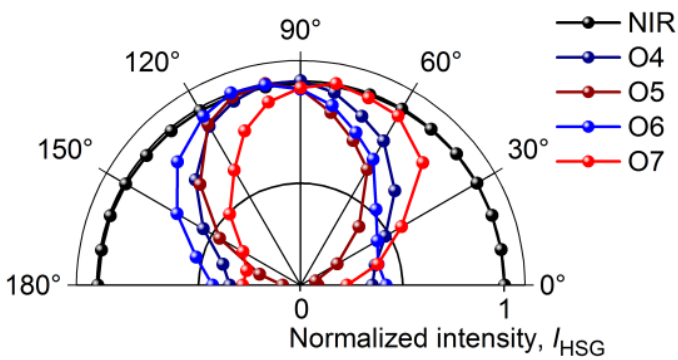

Fig. 2. a High-order sideband intensity $I_{\mathrm{HSG}}$ (multiplied with indicated factors) in the polarization basis parallel $\left(\theta=0^{\circ}\right.$, light colors) and perpendicular $\left(\theta=0^{\circ}\right.$, dark colors) to the driving field. Even and odd orders are cross-polarized. b For a selective excitation at the $\mathrm{K}$ valley with a circularlypolarized preparation pulse (black curve), the intense multi- $\mathrm{THz}$ fields drive electron-hole pairs into the non-excited K' valley adding polarization components of opposite helicity which causes the emission of elliptically polarized sidebands (normalized, colored data points).

symmetry for positive and negative field polarities and enables the generation of odd sideband orders. As a direct consequence of the valley pseudospin, straightforward vector algebra shows that even orders have to be polarized parallel to the driving field, while the odd orders exhibit a perpendicular polarization. The polarization-resolved sideband intensity $I_{\mathrm{HSG}}$ (Fig. 2a) confirms this conjecture and shows cross-polarized even and odd orders. This measurement underlines the direct link between odd-order sidebands and the valley pseudospin in monolayer $\mathrm{WSe}_{2}$ and proves that the valley coherence is conserved even under atomically strong fields.

In order to directly trace how the valley pseudospin changes during lightwave acceleration, we prepare excitons selectively in the $\mathrm{K}$ valley by a right-circularly polarized excitation pulse (Fig. 2b, black curve). If the electron-hole pairs recollide within the same valley, the sideband emission is expected to be polarized with the same helicity as the excitation pulse. Surprisingly, the emitted sidebands of order $n=4$ to 7 (Fig. 2b, colored data points) are polarized strongly elliptically with a predominant vertical orientation, which is in stark contrast to the circularly polarized preparation pulse. The overall elliptical polarization stems from counter-circularly polarized contributions of the non-excited $\mathrm{K}$ ' valley due to lightwave-driven intervalley transport. Here, the intense $\mathrm{THz}$ waveforms shift a sizable amount of photoexcited electron-hole pairs adiabatically into the opposite valley.

In conclusion, we showed that lightwave acceleration can not only change the translational motion of charge carriers, but even control internal quantum attributes such as the valley pseudospin. In detail, the valley pseudospin in monolayers of $\mathrm{WSe}_{2}$ manifests itself in a qualitatively new signature - odd-order sidebands. We introduced this new paradigm by demonstrating lightwave-driven intervalley transport opening a new playground for ultimately fast room-temperature valleytronics at optical cycle scales.

1. P. B. Corkum, F. Krausz, Nature Phys. 3, 381-387 (2007).

2. S. Ghimire et al., Nature Phys. 7, 138-141 (2011).

3. O. Schubert et al., Nature Photon. 8, 119-123 (2014).

4. H. Liu et al., Nature Phys. 13, 262-265 (2017).

5. M. Hohenleutner et al., Nature 523, 572-575 (2015).

6. B. Zaks, R.B. Liu, M. Sherwin, Nature 483, 580-583 (2012).

7. F. Langer et al., Nature 533, 225-229 (2016).

8. X. Xu, W. Yao, D. Xiao, T. F. Heinz, Nature Phys. 10, 343-350 (2014).

9. F. Langer et al., Nature 557, 76-80 (2018).

10. A. M. Jones et al., Nature Nanotechnol. 8, 634-638 (2013). 